\title{
Clinical presentation and management of neonatal abstinence syndrome: an update
}

This article was published in the following Dove Press journal:

Research and Reports in Neonatology

9 April 2014

Number of times this article has been viewed

\author{
Alice Ordean' \\ Brian C Chisamore ${ }^{2}$ \\ 'Department of Family Medicine, \\ ${ }^{2}$ Department of Pediatrics, St Joseph's \\ Health Centre, and University of \\ Toronto, Toronto, ON, Canada
}

\begin{abstract}
Exposure to prescription medications and illicit drug use during pregnancy has been associated with neonatal abstinence syndrome. The clinical presentation consists of neurological respiratory, gastrointestinal, and vasomotor disturbances. All infants require observation and supportive care to ensure appropriate adaptation and growth in the newborn period. A smaller percentage may also require additional pharmacotherapy, depending on the specific gestational substance exposure. Women should be counseled antenatally about the possible neonatal effects, and mother-baby dyad care should be implemented for this particular patient population.

Keywords: neonatal withdrawal, opioids, marijuana, cocaine, benzodiazepines, selective
\end{abstract} serotonin reuptake inhibitors

\section{Introduction}

Maternal substance use can lead to adverse neonatal outcomes, including neonatal withdrawal, as well as neonatal presentations consistent with acute toxicity or lasting drug effects. ${ }^{1}$ Neonatal abstinence syndrome (NAS) is a constellation of withdrawal symptoms and signs exhibited by newborns in response to discontinuation of gestational exposure to various substances. ${ }^{1}$ Neonatal withdrawal presents with symptoms that increase over time due to lack of drug exposure and lowering drug levels. In contrast, neonatal toxicity represents the drug effect due to in utero exposure, and symptoms typically improve over time as drug levels decrease. NAS has been reported with gestational exposure to both prescription medications, such as opioids and benzodiazepines, and illicit drugs. ${ }^{1}$

The incidence of NAS is related to rates of maternal substance use. Estimates of the prevalence of substance use during pregnancy depend on the screening method used and the geographic location. According to the 2010 National Survey on Drug Use and Health, 4\%-5\% of pregnant women aged 15-44 years were current illicit drug users (including marijuana, cocaine, heroin, and nonmedical use of prescription drugs such as pain relievers, tranquilizers, or sedatives). ${ }^{2}$ The rate was higher among pregnant women younger than 25 years of age (16\% among those aged 15-17 years and 7\% among those aged 18-25 years) in comparison with those aged 26-44 years (approximately 2\%). Other estimates based on urine toxicology screening (including for benzodiazepine, marijuana, cocaine, opiates, amphetamines, phencyclidine, and barbiturates) of pregnant women on admission for delivery at a New Orleans Universal Hospital found that $19 \%$ of that population screened positive for at least one substance. ${ }^{3}$ In contrast with the other study, women who used illicit substances were older and
Correspondence: Alice Ordean

St Joseph's Health Centre,

30 The Queensway, Toronto,

ON M6R IB5, Canada

$\mathrm{Tel}+\mathrm{I} 4165306860$

$\mathrm{Fax}+\mathrm{I} 4165306160$

Email ordeaa@stjoe.on.ca 
had higher parity. These statistics indicate a growing need for more detailed screening of infants for NAS and offering appropriate clinical management on postpartum and neonatal wards. This article provides an evidence-based clinical review of the presentation and management of NAS secondary to in utero exposure to opioids and benzodiazepines, as well as adverse neonatal effects secondary to maternal use of marijuana, cocaine, and selective serotonin reuptake inhibitors (SSRIs).

\section{Opioids}

Opioids encompass a class of natural, endogenous, and synthetic compounds that primarily activate the mu receptor. The term "opiate" refers to a class of alkaloid compounds derived naturally from the poppy, such as morphine and codeine. Heroin, oxycodone, hydromorphone, methadone, and buprenorphine are examples of synthetic opioids. ${ }^{4}$ The main clinical uses of opiates include analgesia and cough suppression in adults.

\section{Prevalence of perinatal opioid use and NAS}

The growing increase in NAS has paralleled the epidemic of maternal misuse of prescription opioids. Based on a retrospective review of a nationally representative US database of births between 2000 and 2009, as the rate of maternal opiate use increased by approximately five-fold from 1.19 to 5.63 per 1,000 hospital births per year, the incidence of NAS almost tripled from 1.20 to 3.39 per 1,000 hospital births per year. ${ }^{5}$ In actual numbers, this means that over 13,000 babies were born with NAS in the USA in 2009. In Canada, the use of opioids has also reached concerning levels. Specifically, Ontario has the highest rate of opioid use in Canada, and has one of the highest rates of prescription opioid use in the world. ${ }^{6}$ In 2009, the incidence of NAS in Ontario increased to $3-4$ cases per 1,000 deliveries. $^{7}$ This growing rate of NAS translates into an estimated $3 \%$ of neonatal intensive care beds at a given time being utilized for these infants, leading to increased health care expenditure due to prolonged hospital admissions for management of NAS. ${ }^{7}$

\section{Management of maternal opioid dependence}

The mainstays of treatment for opioid dependence during pregnancy are opioid agonists. Pharmacotherapy options include methadone and buprenorphine preparations. Methadone has been endorsed until recently as the standard of care for the management of maternal opioid use disorder based on prolonged clinical experience and its ability to enhance retention in care and to improve maternal and neonatal outcomes. $^{6-8}$

Buprenorphine has been investigated as an alternative treatment because of its potential to reduce the severity of NAS. The MOTHER (Maternal Opioid Treatment: Human Experimental Research) study was a double-dummy, doubleblind, randomized controlled trial that compared methadone and buprenorphine maintenance for the management of opioid dependence during pregnancy. ${ }^{9}$ This study confirmed the role of methadone in treating pregnant women and provided evidence to support buprenorphine as an alternative to methadone. Buprenorphine was found to have a higher dropout rate, likely due to higher patient dissatisfaction with this medication; however, buprenorphine was associated with reduced severity of NAS.

Due to the lack of convenient access to buprenorphine, some pregnant women may be maintained on combination treatment of buprenorphine + naloxone. Debelak et al published some initial results for ten pregnant women treated with buprenorphine + naloxone (eight were receiving this medication preconception and two were initiated during the first trimester). ${ }^{10}$ Most neonates were delivered at term with normal birth parameters. Treatment for NAS was required by $40 \%$ of infants, and the mean neonatal length of stay was comparable with previous findings with buprenorphine and methadone treatments. ${ }^{11}$ Similar findings were reported by Wiegand et al, who demonstrated that use of the combined buprenorphine + naloxone product was associated with a lower incidence of NAS and a shorter duration of hospital stay. ${ }^{12}$ Use of buprenorphine + naloxone during pregnancy was not associated with any significant negative maternal or neonatal outcomes. Further research is required before the safety of this combination product can be determined. Until data become available, pregnant opioid-dependent women should be offered maintenance treatment with methadone or buprenorphine monoproduct.

\section{Presentation of opioid withdrawal}

\section{Clinical symptoms/signs and pathophysiology}

Pregnant women using any opioid on a regular basis risk having a newborn with NAS. The incidence of NAS secondary to opioids is as high as $85 \% .{ }^{8}$ Neonatal opioid withdrawal is characterized by neurological, gastrointestinal, and autonomic manifestations. ${ }^{13}$ Signs include increased muscle tone, tremor, high-pitched cry, poor feeding, poor weight gain, diarrhea, temperature instability, nasal stuffiness, and irritability. Seizures have also been described in $2 \%-11 \%$ 
of untreated newborns withdrawing from opioids, including both heroin and methadone. ${ }^{1,14,15}$ Abnormal electroencephalograms have been described in as many as one third of withdrawing neonates, with normalization by $6-12$ months of life. ${ }^{15}$ Reported seizures tend to be generalized motor seizures and myoclonic jerks.

The pathophysiology for NAS is related to the agonist effects of opioids that produce supraspinal analgesia, sedation, euphoria, respiratory depression, and decreased gastrointestinal motility. ${ }^{1,13}$ At a cellular level, opioids acutely inhibit the release of noradrenaline at synaptic terminals. With chronic opioid exposure, tolerance develops as the rate of noradrenaline release from the synapse increases towards normal. Abrupt discontinuation of opioids results in supranormal release of noradrenaline, and produces the autonomic and behavioral signs of withdrawal. ${ }^{1}$ Given that the mu receptors are concentrated mostly in tissues of the gastrointestinal and central nervous system, it is expected that characteristic signs of withdrawal would include central nervous system excitability, autonomic over-reactivity, and gastrointestinal dysfunction.

The onset of clinical symptoms and signs of NAS depends on the half-life of the opioid used. Onset of symptoms of NAS in neonates exposed to maternal heroin and prescription opioids typically occurs within 24 hours of birth, whereas withdrawal from methadone and buprenorphine usually commences around 48-72 hours of age, with late presentations up to 5-7 days after birth. ${ }^{1,8}$ The NAS associated with buprenorphine appears to start within 12-48 hours, peaks within 72-96 hours, and lasts for 120-168 hours. ${ }^{16}$

The NAS profile has been shown to differ between methadone-treated and buprenorphine-treated infants. Secondary analyses from the MOTHER study showed that the mean total NAS score was significantly higher in methadone-exposed infants in comparison with their buprenorphine-exposed counterparts. ${ }^{17}$ This difference was due to greater severity for five of the 19 (26\%) individual NAS signs, including central nervous system hyperirritability (eg, hyperactive Moro reflex, disturbed and undisturbed tremors, excessive irritability) and failure to thrive. A higher mean score for sneezing was demonstrated among buprenorphine-exposed neonates. In addition, nasal stuffiness and loose stools were often observed with buprenorphine. This trial also found a significant difference in median time to initiation of treatment of 36 hours for methadone-exposed neonates versus 59 hours for buprenorphine-exposed neonates. These findings were hypothesized to exist due to variations in transplacental transfer and the pharmacokinetics of these medications.

The severity of NAS can be affected by several variables. Results from the MOTHER study indicated that higher infant birth weight and nicotine exposure (ie, number of cigarettes smoked 24 hours prior to delivery) were associated with an increased likelihood of requiring treatment for NAS. ${ }^{18}$ Nicotine exposure also predicted a higher total dose of medication required to treat NAS, but did not lead to higher NAS scores or longer treatment. These findings replicated earlier observations demonstrating that heavy smoking (defined as $\geq 20$ cigarettes/day), was associated with higher cumulative Finnegan score, higher daily Finnegan score, and longer hospital stay. ${ }^{19}$

Maternal use of SSRIs, antidepressants, and antipsychotics was associated with longer treatment for NAS, as well as a higher total dose of morphine required to treat NAS. Maternal use of anxiolytics failed to show any relationship with any of these outcome variables. This result is in contrast with previous studies documenting that maternal use of benzodiazepines in addition to methadone was associated with an increased length of stay, whereas SSRIs failed to have any effect. ${ }^{20}$

Historically, studies have reported contradictory results concerning the relationship between maternal methadone dose and severity of NAS. Cleary et al performed a systematic review and meta-analysis to determine if there is evidence confirming an association between these two factors. ${ }^{21}$ Sixtyseven studies were included in their review, with 29 in the final meta-analysis. The authors concluded that the severity of NAS was not related to low-dose or high-dose methadone. The relationship between buprenorphine dose and NAS severity remains unconfirmed. ${ }^{22}$ This finding was replicated in the MOTHER study, indicating that maternal medication dose (methadone or buprenorphine) at delivery does not have any relationship with NAS. ${ }^{18}$ Jones et al published further evidence in 2014 showing that maternal buprenorphine dose was not related to any of ten neonatal clinical outcomes, including severity of NAS, total amount of morphine used to treat NAS, duration of treatment for NAS, or length of hospital stay. ${ }^{23}$

Evidence from the past decade seems to confirm an earlier finding that premature infants tend to exhibit less severe NAS than term infants. ${ }^{24,25}$ In comparison with preterm infants, term infants required a longer length of stay and a longer treatment duration, as well as higher morphine doses to treat symptoms of NAS. This apparently decreased severity of signs of NAS in preterm infants could be explained 
by the following: objective withdrawal tools for NAS were designed for term infants, immature development of the central nervous system, reduced total exposure time to opioids, and reduced placental transfer rate. ${ }^{24}$

\section{Differential diagnosis}

Opioid-related NAS may mimic other conditions. ${ }^{1,13}$ No clinical signs should be solely attributed to drug withdrawal without a careful assessment to exclude other causes. Therefore, it is important to rule out sepsis, hypoglycemia, hypocalcemia, hyperthyroidism, intracranial hemorrhage, hypoxic ischemic encephalopathy, and polycythemia/hyperviscosity syndrome.

\section{Management of the neonate}

\section{Assessment for NAS}

Several screening tools have been developed, including the Finnegan neonatal abstinence scoring system, the Lipsitz neonatal drug-withdrawal scoring system, the Ostrea tool, the Neonatal Withdrawal Inventory, and the Neonatal Narcotic Withdrawal Index. ${ }^{13,26}$ The Finnegan neonatal abstinence scoring system was developed in 1975 by Loretta Finnegan to provide a standardized method for assessing drug-exposed infants. The score was based on the 20 most commonly found clinical symptoms and signs of neonatal narcotic withdrawal, and consisted of 31 parameters which were further reduced to 21 items. ${ }^{27,28}$ Based on a survey of neonatal-perinatal programs across the USA and Europe, some form of the Finnegan scoring tool remains the most commonly used ${ }^{29,30}$ and best validated ${ }^{6,26,31}$ scoring tool.

The Lipsitz neonatal drug-withdrawal scoring system was the second most commonly used scoring tool across the USA. ${ }^{29}$ This tool consists of eleven items which are scored from 0 to 3 based on severity at presentation, with four signs being rated as only yes/no. ${ }^{32}$ The Lipsitz tool has a reported sensitivity of $77 \%$ based on a total score $>4$ indicating significant withdrawal. No further validation studies have been conducted and/or published in the literature since its inception.

The MOTHER study proposed a revised Finnegan scoring tool by making further modifications, including taking out items that overlapped with others (eg, frantic sucking of fists is related to hypertonicity) or were not responsive to therapy (eg, myoclonic jerking or mottling), and consolidating items (eg, regurgitation and projectile vomiting, watery/loose stools). Two items, specifically irritability and failure to thrive, were also added to score. ${ }^{33}$ This version of the modified Finnegan scoring, named the MOTHER NAS scale, consisted of 19 signs and symptoms that were used for scoring and medication decision-making. Inter-rater reliability testing was performed throughout the study period and demonstrated a high level of agreement between expert rater and site raters. ${ }^{9}$ This score is intended to be implemented every 3 hours and to reflect the infant's status over this period of observation.

Based on several guidelines, assessment of NAS should be performed using an objective NAS scoring tool to determine the severity of withdrawal and the need for initiation of pharmacologic treatment. ${ }^{1,6,7,13}$ Scoring should be initiated within hours of life and continued for 72 hours (for opioids with a short half-life) or up to 120 hours (for opioids with a longer half-life) to assess for possible neonatal withdrawal symptoms and signs.

\section{Pharmacologic management}

When scores reach a threshold despite adequate use of nonpharmacologic strategies, pharmacologic treatment is recommended. ${ }^{6,7,13,26}$ Treatment for NAS may be indicated in up to $40 \%-50 \%$ of infants. ${ }^{1}$ Data from the MOTHER study indicated that $41 \%$ of neonates exposed to methadone in comparison with $27 \%$ of those exposed to buprenorphine required pharmacotherapy for NAS, and these rates were not significantly different. ${ }^{9}$ Use of a cardiac monitor is recommended from the initiation of morphine for a period of 4 days and/or until the pharmacotherapy dose is to be decreased. ${ }^{6,7}$ Weaning off pharmacotherapy should commence after neonatal withdrawal symptoms have been stabilized. Weaning protocols tend to be center-specific. ${ }^{1}$ Scoring should continue until 48-72 hours after discontinuation of pharmacologic therapy.

There are two different strategies used for treatment of NAS, ie, weight-based, where medication is dispensed on a $\mathrm{mg} / \mathrm{kg}$ basis, and symptom-based, where dosing is weightindependent and determined by NAS score. ${ }^{26}$ These two regimens vary in terms of the recommended initial dose, dose increments, weaning dose, and initiation of adjunctive treatment. No standardized approach has been determined due to lack of comparative studies.

\section{Opioids as initial treatment for NAS}

Numerous pharmacotherapy options have been considered for management of NAS secondary to opioid withdrawal. Based on a Cochrane review, the American Academy of Pediatrics guideline, and other expert reviews, opioids such as morphine are recommended as initial treatment for NAS. ${ }^{26,34-36}$ Opioids have been shown to reduce the time taken to regain birth weight and duration of supportive care. In comparison with 
phenobarbital, opioids decrease the incidence of severe NAS (eg, seizures) and reduce the treatment duration and rate of admission to nursery. Therefore, opioids were determined to be first-line agents for the management of NAS.

Morphine is the most commonly used opioid in the management of NAS, as recommended by the American Academy of Pediatrics guideline and also based on surveys conducted in the USA and Europe. ${ }^{1,29,30}$ Oral morphine solutions contain no alcohol or additives, so are ideal for neonatal administration. Morphine preparations include diluted, deodorized tincture of opium, and preservative-free morphine hydrochloride solution.

Methadone represents an alternative to morphine. Based on national surveys, methadone is used infrequently in the treatment of NAS. ${ }^{29,30}$ Depending on geographic location, there is an up to $20 \%$ prevalence of methadone use in the management of NAS. A retrospective chart review compared length of stay and other parameters for methadone versus oral morphine preparations for the treatment of NAS. ${ }^{37}$ There were no significant differences in gestational age, birth parameters, or length of stay between the two treatment groups.

Buprenorphine has also been examined recently as an alternative pharmacotherapy. The first pilot study consisted of a randomized, open-label trial of sublingual buprenorphine compared with oral morphine solution. ${ }^{38}$ Sublingual buprenorphine resulted in a $31 \%$ reduction in length of treatment (27 days for buprenorphine versus 22 days for morphine) and a $29 \%$ reduction in length of hospital stay (32 days for buprenorphine versus 22 days for morphine), but neither difference reached statistical significance. A second, randomized, open-label trial designed as a Phase I safety study compared sublingual buprenorphine with oral morphine in the management of NAS. ${ }^{39}$ The findings included a statistically significant $24 \%$ reduction in duration of hospital admission in the buprenorphine group (from 42 to 32 days), and a $40 \%$ reduction in length of treatment ( 23 days for buprenorphine versus 38 days for morphine). These two preliminary reports indicate that buprenorphine may be a safe alternative medication in the treatment of neonatal withdrawal; however, larger double-blind studies are required to validate these results. The optimal pharmacotherapeutic agent for NAS has yet to be determined.

\section{Co-use of clonidine}

There is promising evidence regarding the benefit of adding a secondary pharmacotherapeutic agent such as clonidine. Agthe et al performed a randomized controlled trial assessing the benefit of clonidine when added to dilute tincture of opium. ${ }^{40}$ The results showed that addition of clonidine shortened the median length of therapy by $27 \%$ (11 days for clonidine versus 15 days for placebo). Treatment failure was reported in $12.5 \%$ of infants treated with placebo in comparison to none in the clonidine group. However, adequate clinical trials are required to establish an efficacious and safe dose. ${ }^{6,7}$ The mechanism of action of clonidine as an agonist is at the central alpha2adrenergic receptor and perhaps at the imidazoline- 1 receptor to reduce sympathetic outflow. Hence it acts to ameliorate tachycardia, hypertension, restlessness, and diarrhea. In cases where high doses of morphine are being used, clonidine may be considered a second-line agent as a medical adjunct to assist in controlling withdrawal symptoms. ${ }^{7,33}$

\section{Co-use of phenobarbital}

Using a barbiturate as a single agent to treat opioid NAS is not generally recommended unless there is a mixed withdrawal syndrome. However, it may be acceptable in the absence of other preparations or expertise to prepare dilute solutions of morphine sulfate. ${ }^{7}$ A Cochrane review published in 2005 indicated that in cases where infants were exposed to maternal polysubstance use, especially opioids in conjunction with sedatives, alcohol, or barbiturates, phenobarbital may reduce the severity of withdrawal and should be used in preference to diazepam to reduce the risk of treatment failure. ${ }^{41}$ More recent data published in 2013 compared the efficacy of clonidine versus phenobarbital in reducing the length of morphine treatment. ${ }^{42}$ Phenobarbital tended to reduce the length of treatment by 4 days (not statistically significant); however, both groups required the same total morphine dose. The overall duration of treatment for NAS was shorter with clonidine since no outpatient tapering was required, whereas infants on phenobarbital continued on this medication on an outpatient basis for up to 8 months. Therefore, the use of morphine in combination with phenobarbital has been reported in the treatment of infants whose symptoms are not well controlled with morphine alone, but strong evidence of its efficacy is lacking.

\section{Outpatient tapering protocol}

Weaning of pharmacotherapy usually occurs in an inpatient setting to allow for careful titration of infant doses due to concerns about the ability of parents to care for infants at home and the lack of adequate follow-up by health care providers for various reasons. However, some institutions are embarking on outpatient treatment in selected patient populations. For example, a survey of neonatal units in the UK and Ireland reported that $29 \%$ of units were found to discharge neonates home on medications. ${ }^{30}$ 
A hospital in Australia developed a hospital-based weekly follow-up clinic in 1998 to provide coordinated care for substance-exposed infants after discharge from hospital. ${ }^{43}$ This study reported that the availability of an outpatient clinic led to a significant reduction in length of stay in hospital and length of admission to their nursery; however, the duration of morphine treatment was significantly longer for infants discharged home on pharmacotherapy (57.5 days) in comparison with infants tapered down on an inpatient basis (17.2 days). Therefore, in a suitable subpopulation, neonates may be discharged home on morphine to undergo a slow tapering process if appropriate follow-up by a multidisciplinary group of health care providers is available.

Another program developed in the USA combined inpatient initiation of methadone treatment followed by a weaning process in the community, which is in contrast with the traditional routine of completing treatment in a neonatal intensive care unit. ${ }^{44}$ Patients were screened to ensure that they met the criteria for methadone weaning on an outpatient basis. The results indicate that the combined group had a shorter length of hospital stay and reduced hospital costs, but a longer duration of methadone treatment despite a similar cumulative methadone dosage. Further research is needed to evaluate the suitability of this approach and its associated long-term benefits.

\section{Future areas of research}

Innovative advances in this field consist of elucidating the role of different pathways in opioid withdrawal and medications for the management of NAS. New areas of research include the role of serotonin in opioid withdrawal, so targeting of serotonergic receptors may reduce opioid withdrawal symptoms in humans. ${ }^{45}$ Alternatively, use of SSRIs to increase the amount of serotonin at the synapse may be another mechanism for reducing opioid withdrawal. Vaccines to target illicit and prescription opioids are also being developed as adjunctive therapies. In addition, pharmacogenomic studies are considering ways to study genes affecting the individual pharmacodynamic and pharmacokinetic parameters of opioids.

One preliminary prospective cohort study addressed the association between genotype for single nucleotide polymorphisms and length of hospital stay, need for medical treatment for NAS, and treatment with two or more medications. ${ }^{46}$ The results indicate that genetic variants were associated with a shortened length of stay, decreased likelihood of treatment, and treatment with multiple medications. Most of these hypotheses require further investigations in humans before definitive evidence is available and application to clinical cases can be considered.

\section{Nonpharmacologic measures}

Nonpharmacologic supportive care is essential for all infants with NAS regardless of the need for pharmacotherapy. Interventions consist of support for the mother-baby dyad, environmental modifications, soothing behaviors, and positional support, as well as frequent, smaller volume, hypercaloric feeds. These are all measures that may be beneficial in the treatment of neonates with NAS, both in hospital and in the home environment. ${ }^{1,7,9,26}$

Adapting the environment for decreased sensory stimulation is beneficial to both mother and baby. ${ }^{7}$ Cuddling, overall gentle handling, skin-to-skin contact (Kangaroo care), and infant slings have all been promoted as ways of improving behavioral adaptation in newborns with NAS. ${ }^{7}$ Watchfulness and reinforcement of self-soothing behaviors used by the neonate (non-nutritive sucking, swaddling) may assist in ameliorating NAS. Minimization of excessive handling, respect for the neonates' sleep state, and environmental stimulation will also help with regulation of the transition between neonatal behavioral states, and thus may also have an impact on NAS. ${ }^{7}$

Specific holding/constraining techniques, proper positioning, use of gentle firm pressure, and gentle vertical rocking can all support the neonate's self-regulation. ${ }^{7}$ Rocking beds or mechanical swings should be used as an adjunct if the care staff feel that these are helpful to reduce withdrawal symptoms. However, there is evidence that, for some neonates, this may in fact be overstimulating during the acute period of withdrawal and may not be appropriate. ${ }^{46,47}$

Frequent, smaller volume, hypercaloric feeds are generally recommended for infants who have feeding difficulties because of regulatory control issues and/or poor weight gain due to excessive caloric expenditure, gastroesophageal reflux, and diarrhea. ${ }^{7}$ Dietitian support is part of the multidisciplinary approach to care of the neonate with NAS. Evolving now are family-integrated neonatal care studies that seem to reduce parental stress, and adaptations to these models for this neonatal population are anticipated soon.

\section{Breastfeeding}

The role of breastfeeding in managing NAS remains uncertain. Some studies have documented a benefit from breastfeeding in terms of reduced pharmacotherapy for NAS, while others have failed to find a similar outcome. Earlier data from a retrospective chart review demonstrated that breast-fed infants 
had significantly lower NAS scores, a lower prevalence of medical treatment for NAS (53\% versus 79\%) and a shorter duration of stay (15 days versus 19 days) compared with a formula-fed group, even after controlling for polysubstance use and prematurity. ${ }^{48}$ However, there was no difference in the mean maximum dose of morphine. There were significant limitations to this study, including breastfeeding mothers being more likely to receive more comprehensive antenatal care, have reduced polydrug use, and being less likely to be involved with child welfare services.

In a more recent publication, a retrospective chart review by McQueen et al corroborated a similar trend of reduced severity and duration of NAS scores in predominantly breast-fed, methadone-exposed infants in comparison with combination-fed or predominantly formula-fed neonates. ${ }^{49}$ Jansson et al compared methadone-maintained women who were breastfeeding with matched formula-feeding women in terms of methadone concentrations and neonatal effects. ${ }^{50}$ Methadone plasma concentrations were not different between the groups and methadone concentrations in breast milk were low. Neurobehavioral assessments did not demonstrate any significant effect of breastfeeding; however, there was a trend towards reduced pharmacotherapy for breast-fed infants. Pritham et al also demonstrated an association between infant feeding and length of stay. ${ }^{51}$ Breastfeeding was linked to lower rates of infant treatment for NAS and shorter length of stay. This association was seen with both methadone and buprenorphine exposures in utero.

Several case reports and small case series documented that the concentrations of buprenorphine and norbuprenorphine detected in breast milk are generally low and did not exceed maternal plasma concentrations ${ }^{52-55}$ Based on further calculations due to its poor bioavailability, infants would receive $<1 \%$ of the maternal weight-adjusted dosage, which is unlikely to lead to any adverse effects in the neonate. However, only a couple of studies addressed the effect of lactation on NAS in buprenorphine-exposed infants. One case report published in 1997 described little pharmacologic effect of buprenorphine detected in breast milk since the baby did not display any withdrawal signs when breastfeeding was abruptly interrupted due to maternal illness at 8 weeks of age. ${ }^{52}$ In a more recently published observational study, $70 \%$ of mothers were reporting to breastfeed while on buprenorphine; however, $35 \%$ of infants still required treatment for NAS, which may indicate that buprenorphine exposure in breast milk does not alter NAS. ${ }^{56}$

Further research into the neonatal and long-term effects of exposure to methadone or buprenorphine in breast milk is required. Based on the current evidence, since breastfeeding itself may provide additional benefits to opioid-exposed infants, mothers should be encouraged to consider breastfeeding if there are no active contraindications.

\section{Illicit drugs Stimulants}

Stimulants consist of both naturally occurring plant alkaloids, such as cocaine, and synthetic compounds, such as amphetamines. ${ }^{4}$ These compounds act to increase monoamine (dopamine, norepinephrine, and serotonin) activity in the central and peripheral nervous systems through various mechanisms.

\section{Presentation}

Earlier studies from the 1980s and 1990s described some characteristic neonatal symptoms and signs related to cocaine exposure in utero. Initial reports of neonatal symptoms secondary to cocaine and methamphetamine exposure consisted of neurologic and physiologic alterations such as abnormal sleep patterns, tremors, hypertonia, high-pitched cry, frantic fist sucking, poor feeding, mild jitteriness, and mild tachypnea. ${ }^{57,58}$ Symptoms peaked on days 2 and 3 and resolved without any treatment. It was unclear if these symptoms were signs of intoxication or withdrawal. Doberczak et al reported the presence of neurobehaviors using a standardized scoring system for clinical assessment of neonatal withdrawal following intrauterine cocaine exposure. ${ }^{59}$ Neonatal symptoms included increased muscle tone, irritability or tremors, and transient electroencephalographic abnormalities, with no respiratory, gastrointestinal, or autonomic dysfunction. Similarly, neurological findings peaked on day 3 and improved without any treatment, which pointed towards a direct neurotoxic effect of cocaine rather than withdrawal, given that symptoms and signs resolved over time.

Other studies have failed to find any characteristic neonatal withdrawal syndrome based on the Brazelton Neonatal Behavioral Assessment Scale. ${ }^{60,61}$ In addition, more recent controlled studies, published in the 2000s, have failed to confirm earlier reports regarding cocaine withdrawal. ${ }^{62,63}$ Eyler et al compared cocaine-exposed infants based on urine toxicology testing status at birth and failed to find any evidence for acute cocaine toxicity or cocaine withdrawal in those infants who were positive for cocaine metabolites at birth. ${ }^{64}$ However, an overall drug effect of cocaine was noted, and consisted of increased autonomic regulation (eg, more startles and tremors). Similarly, the Maternal Lifestyle Study failed to demonstrate neonatal withdrawal in cocaine-exposed infants but did report mild, transient neurologic findings such 
as irritability, jitteriness, tremors, high-pitched cry, and excessive sucking. ${ }^{65}$ Investigators concluded that these increased central and autonomic nervous system symptoms and signs were more likely in keeping with a cocaine effect. Therefore, due to the lack of convincing evidence from controlled studies, neonatal cocaine withdrawal is not likely a true syndrome and earlier findings may have been more indicative of an intrauterine cocaine exposure effect.

\section{Management}

Infants born to mothers using stimulants during pregnancy require only supportive care. There is no evidence about the need or use of pharmacotherapeutic interventions.

\section{Cannabis}

Cannabis preparations consist of marijuana, hashish, and hash oil. ${ }^{4}$ The major psychoactive component in marijuana is delta9-tetrahydrocannabinol, which acts on cannabinoid receptors in the central nervous system. At low doses, marijuana leads to a mixture of depression and stimulation; however, central nervous system depression is more common at higher doses of marijuana.

\section{Presentation}

Two longitudinal cohort studies have documented neonatal neurobehavioral changes associated with marijuana use during pregnancy. The Ottawa Prenatal Prospective Study found that prenatal exposure to marijuana was associated with increased tremors and exaggerated startles, both spontaneous and those provoked by external stimuli, as well as poorer habituation to visual stimuli based on the Brazelton Neonatal Assessment Scale assessment at 3 days of age. ${ }^{66}$ A follow-up study of this population at 9 and 30 days of age demonstrated persistent neurological findings. ${ }^{67}$ The continued presence of symptoms suggested that the findings may be attributable to marijuana-related withdrawal. However, given that tetrahydrocannabinol is highly lipophilic, these prolonged clinical signs may be also be indicative of a marijuana drug effect.

The Maternal Health Practices and Child Development Study reported on prenatal use of marijuana in a high-risk population of women in the USA. Findings included autonomic arousal, such as increased high-pitched crying and sleep disturbances. ${ }^{68,69}$ Neurobehavioral effects as documented by the Ottawa Prenatal Prospective Study were not found in this study, and use of marijuana was not predictive of neonatal behaviors. ${ }^{70}$ Therefore, the evidence regarding the presence of a neonatal marijuana withdrawal syndrome is conflicting, and further studies are required to clarify the association between gestational marijuana and documented neurobehaviors.

\section{Management}

Management of neonatal neurobehaviors consists exclusively of supportive care with close monitoring for growth and development.

\section{Prescription medications Benzodiazepines}

The prevalence of anxiety disorders during pregnancy and the postnatal period has not been studied as extensively. Based on a systematic review of the literature by Ross and McLean, obsessive-compulsive disorder and generalized anxiety disorder have high perinatal prevalence rates, being up to $4 \%$ and $9 \%$, respectively. ${ }^{71}$ Perinatal anxiety disorders are associated with adverse obstetric consequences, such as premature labor and delivery, and adverse developmental outcomes due to fetal exposure to elevated hormone levels (eg, cortisol). Pharmacotherapy for anxiety disorders consists of benzodiazepines and SSRIs.

Benzodiazepines act on the gamma aminobutyric acid receptor, leading to sedative, anticonvulsant, and hypnotic effects. ${ }^{4}$ This class of medication has various subgroups with varying durations of action, ie, long-acting (eg, diazepam), intermediate-acting (eg, clonazepam, lorazepam), and shortacting (eg, alprazolam, midazolam) compounds. ${ }^{72}$

\section{Presentation}

Intoxication and withdrawal syndromes related to maternal benzodiazepine use have been reported in the literature. ${ }^{73}$ Laegreid et al compared the effect of maternal use of benzodiazepines in 17 newborn infants with that in a group of 21 newborns exposed to other psychotropic drugs and 29 control newborns with no known exposures. ${ }^{74}$ Neurological examinations demonstrated significant differences, from depression to hyperexcitability of the central nervous system. Symptoms such as drowsiness, absent reflexes, and poor sucking were attributed to neonatal intoxication, whereas other symptoms and signs such as regurgitation, jitteriness, tremor, startles, and monotonous cry were linked to neonatal withdrawal. This study provided evidence for the presence of both a neonatal drug effect and a withdrawal syndrome in response to gestational benzodiazepine use.

Neonatal withdrawal has been linked to more prolonged gestational use of benzodiazepines, but has also been described subsequent to acute maternal use during labor. An early case 
report by Rementeria and Bhatt from 1977 described early onset of symptoms within 2-3 hours after birth, consisting of mild tremor in three infants with prolonged intrauterine exposure to diazepam. ${ }^{75}$ Later onset of withdrawal was manifested by irritability, hypertonicity, tachypnea, vigorous sucking, hyperactivity, vomiting, loose stools, and poor weight gain. Symptoms increased in severity and lasted for variable lengths of time of up to 1 month in duration. A randomized controlled trial of the use of lorazepam premedication for management of labor pain demonstrated some differences on the neurobehavioral examination performed at 2-4 hours and again at 24 hours after birth. ${ }^{76}$ Although no significant differences were found, there was a trend towards poorer results on tests of resistance to passive movement (eg, truncal and general body tone) and more neonatal respiratory depression in infants acutely exposed to lorazepam. Repeat examination at 24 hours showed significant improvement in symptoms such as increased muscle tone and alertness. Therefore, the authors concluded that a single maternal dose of lorazepam is not likely to lead to prolonged withdrawal, such as prolonged respiratory depression.

These findings can be explained by the limited ability of infants to metabolize diazepam, leading to a prolonged presence of the drug in their tissues and, therefore, more prolonged pharmacologic effects, resulting in later onset of withdrawal symptoms/signs at 2-3 weeks of life. ${ }^{75,77}$ Earlier withdrawal symptoms can be attributed to maternal abstinence from benzodiazepine use for a couple of days before delivery.

In summary, there is a spectrum of clinical presentations related to both neonatal intoxication and withdrawal. Neonatal benzodiazepine withdrawal includes hypertonia, hypothermia, hyperbilirubinemia, irritability, abnormal sleep patterns, tremors, suckling difficulties, inconsolable crying, and respiratory depression, with onset immediately after delivery and potentially lasting for up to 3 weeks postnatally. ${ }^{72,78}$

\section{Management}

Neonatal benzodiazepine withdrawal symptoms resemble those of opioid-related NAS; therefore, ruling out narcotic coingestion is important when addressing clinical management. Treatment with phenobarbital has been described in the literature. ${ }^{75}$ Dosing protocols consisted of an initial $3-5 \mathrm{mg} / \mathrm{kg} /$ day of phenobarbital in divided doses. Indications for treatment included increased severity of neonatal withdrawal symptoms and signs and failure to thrive. Most infants required 5-10 mg/ $\mathrm{kg} /$ day for symptom control. Phenobarbital was tapered depending on symptoms by reducing the dose every 3-4 days.

\section{SSRIs}

The prevalence of maternal perinatal depression has a point prevalence of up to $13 \%$ during pregnancy and in the first postpartum year. ${ }^{79}$ Perinatal depression is defined as a major depressive episode occurring either during pregnancy or in the post-partum period. Numerous adverse consequences of maternal mood disorders have been identified, such as increased risk of maternal suicide, decreased maternal engagement and attachment with the infant, as well as increased rates of poor obstetric outcomes. ${ }^{79,80}$ The lifetime version of the Edinburgh Postnatal Depression Scale study reported that $40 \%$ of women with a major depressive disorder developed maternal perinatal depression, and the recurrence rate for postpartum depression was estimated at $25 \% .{ }^{81}$ In addition, further analysis found that women diagnosed with maternal perinatal depression had a more severe and chronic clinical course, consisting of increased functional impairment, and higher rates of professional help and treatment. Risk factors for maternal perinatal depression include a family history of major depressive disorder, personal history of anxiety disorder, childhood trauma, childhood sexual abuse, and history of sexual abuse after the age of 16 years. ${ }^{81}$

SSRIs are a common class of antidepressants for the management of perinatal depression. Medications in this class included fluoxetine, paroxetine, sertraline, fluvoxamine, citalopram, and escitalopram. The prevalence of SSRI use is highly variable. A national cohort of 1,106,757 Medicaid-enrolled women in the USA between 2000 and 2007 found that $20 \%$ of women had a documented diagnosis of depression or other mental health problem. ${ }^{80}$ Overall, $8 \%$ of women were dispensed an antidepressant during pregnancy, with approximately $60 \%$ on medication during the 3 months before conception. The other $41 \%$ initiated pharmacotherapy during pregnancy. Most of these women $(80 \%)$ were treated with SSRIs, indicating an approximately $5 \%$ prevalence of SSRI use during pregnancy. Older women ( $\geq 30$ years of age) and white women had higher rates of antidepressant use. A similar prevalence rate was reported by a multisite study from the Health Maintenance Organization Research Network Center for Education and Research on Therapeutics. ${ }^{82}$ SSRIs were the most commonly dispensed antidepressants in 5.6\% of deliveries during 2001-2005. This number represented a four-fold increase in the use of these medications between 1996 and 2005. Data from the National Birth Defects Prevention Study also showed a significant increase in reported SSRI use from $1.7 \%$ in 1998 to $6.1 \%$ in $2005 .{ }^{83}$ This literature review demonstrates that SSRI use has become more common over the past decade in the 
management of maternal perinatal depression. Therefore, studying the possible effects of SSRI use during pregnancy on the fetus and neonate is critical in managing maternal perinatal depression.

\section{Presentation}

Neonatal effects of in utero exposure to SSRIs, especially in the second half of pregnancy, is concerning due to the risk of neonatal adaptation syndrome and persistent pulmonary hypertension of the newborn. A systematic review has confirmed an association between the use of SSRIs in late pregnancy (ie, $>20$ weeks' gestational age) and the presence of a neonatal adaptation syndrome. ${ }^{84}$ A prevalence rate of $10 \%-30 \%$ has been reported. Manifestations include respiratory, motor, central nervous system, and gastrointestinal symptoms. Infants may exhibit nervousness, sleeplessness, tremor, difficulty eating, respiratory distress, and even seizures. ${ }^{85}$ The clinical presentation is similar to other withdrawal syndromes, and consists of mild-to-moderate signs with onset within hours of birth and resolution in 1-2 days. Neonatal convulsions have been described as a more severe form of withdrawal. ${ }^{86}$ These benign seizures were also associated with SSRI use in the third trimester. Seizures were diagnosed within 14 days of birth and were not associated with serious adverse outcomes. One additional infant convulsion can be expected for every 117 women who are using an SSRI in the third trimester.

Pulmonary hypertension of the newborn has been defined as a failure of the normal relaxation of the pulmonary vasculature during transition after birth, with a 5\%-10\% mortality rate. ${ }^{85,87}$ The association between gestational SSRI exposure and pulmonary hypertension of the newborn was initially suggested by Chambers et al in $2006 .{ }^{88}$ However, other studies have failed to confirm this association. ${ }^{89}$ A systematic review of the literature by Jong et al published in 2012 did confirm the presence of a small increased risk of pulmonary hypertension of the newborn. ${ }^{87}$ The risk of this condition is estimated at $<1 \%$, meaning that $99 \%$ of women exposed to an SSRI in late pregnancy will have a healthy infant. Most cases were described as self-limiting and only required admission to the neonatal intensive care unit. ${ }^{85}$

Given the potential for neonatal effects, a risk-benefit analysis needs to be considered when prescribing SSRIs for the management of maternal perinatal depression. Women need to be counseled about the risks associated with exposure in late pregnancy, which should be weighed against the risks of untreated maternal depression.

\section{Management}

Due to the potential for neonatal adaptation difficulties, it is recommended that babies with late-trimester SSRI exposure be observed in hospital for a minimum of 2 days. ${ }^{90}$ Education should be provided to families on the possible effects of gestational SSRI use and the need for observation in the immediate postpartum period. Alternative causes for symptoms, such as coinfections and concomitant substance use, need to be ruled out.

\section{Conclusion}

Based on multiple reports, maternal use of psychoactive medications and illicit drugs is highly prevalent during pregnancy. This in turn has led to growing numbers of infants presenting with NAS. Well documented NAS has been reported secondary to gestational opioid and psychoactive medication (benzodiazepines, SSRIs) exposure. However, there is a lack of evidence confirming the presence of NAS following gestational cocaine and marijuana exposure. The risk of NAS should be discussed with pregnant and postnatal women to ensure early identification and treatment of infants with NAS. Current knowledge gaps exist, and further research is required to identify the most appropriate and effective assessment tools and management protocols.

\section{Disclosure}

The authors report no conflicts of interest in this work.

\section{References}

1. Hudak ML, Tan RC; The Committee on Drugs and the Committee on Fetus and Newborn. Neonatal drug withdrawal. Pediatrics. 2012;129: e540-e560.

2. Substance Abuse and Mental Health Services Administration. Results from the 2010 National Survey on Drug Use and Health: Summary of National Findings, NSDUH Series H-41, HHS Publication No (SMA) 11-4658. Rockville, MD, USA: Substance Abuse and Mental Health Services Administration; 2011. Available from: http://www.samhsa.gov/ data/NSDUH/2k10ResultsRev/NSDUHresultsRev2010.htm. Accessed February 14, 2014.

3. Azadi A, Dildy GA. Universal screening for substance abuse at the time of parturition. Am J Obstet Gynecol. 2008;198:e30-e32.

4. Ries RK, Fiellin DA, Miller SC, Saitz R, editors. Principles of Addiction Medicine. 4th ed. Philadelphia, PA, USA: Lippincott Williams \& Wilkin; 2009.

5. Hayes MJ, Brown MS. Epidemic of prescription opiate abuse and neonatal abstinence. JAMA. 2012;307:1934-1940.

6. Dow K, Ordean A, Murphy-Oikonen J, et al; Neonatal Abstinence Syndrome Work Group. Neonatal abstinence syndrome clinical practice guidelines in Ontario. J Popul Ther Clin Pharmacol. 2012;19: e488-e506.

7. Maternal-Newborn Advisory Committee Neonatal Abstinence Syndrome Work Group. Neonatal abstinence syndrome (NAS) Clinical practice guidelines. Toronto, Canada: Provincial Council for Maternal and Child Health; 2011. Available from: http://pcmch.on.ca/initiatives/neonatalabstinence-syndrome. Accessed February 14, 2014. 
8. Selby P, Kahan M, editors. Methadone Maintenance: A Physician's Guide to Treatment. 2nd ed. Toronto, Canada: Centre for Addiction and Mental Health; 2011.

9. Jones HE, Kaltenbach K, Heil SH, et al. Neonatal abstinence syndrome after methadone or buprenorphine exposure. N Engl J Med. 2010;363: 2320-2331.

10. Debelak K, Morrone WR, O'Grady KE, Jones HE. Buprenorphine + naloxone in the treatment of opioid dependence during pregnancy initial patient care and outcome data. Am J Addict. 2013;22:252-254.

11. Lund IO, Fischer G, Welle-Strands GK, et al. A comparison of buprenorphine + naloxone to buprenorphine and methadone in the treatment of opioid dependence during pregnancy: maternal and neonatal outcomes. Subst Abuse. 2013;7:61-74.

12. Wiegand S, Stringer E, Seashore C, et al. Buprenorphine/naloxone $(B / N)$ and methadone (M) maintenance during pregnancy: a chart review and comparison of maternal and neonatal outcomes. Am J Obstet Gynecol. 2014;210:S368-S369.

13. Bio LL, Siu A, Poon CY. Update on the pharmacologic management of neonatal abstinence syndrome. J Perinatol. 2011;31:692-701.

14. Herzlinger RA, Kandall SR, Vaughan HG Jr. Neonatal seizures associated with narcotic withdrawal. J Pediatr. 1977;91:638-641.

15. van Baar AL, Fleury P, Soepatmi S, Ultee CA, Wesselman PJ. Neonatal behavior after drug dependent pregnancy. Arch Dis Child. 1989;64 235-240.

16. Johnson RE, Jones HE, Fischer G. Use of buprenorphine in pregnancy: patient management and effects on the neonate. Drug Alcohol Depend. 2003;70:S87-S101.

17. Gaalema DE, Scott TL, Heil SH, et al. Differences in the profile of neonatal abstinence syndrome signs in methadone- versus buprenorphine-exposed neonates. Addiction. 2012;107 Suppl 1:53-62.

18. Kaltenback K, Holbrook AM, Coyle MG, et al. Predicting treatment for neonatal abstinence syndrome in infants born to women maintained on opioid agonist medication. Addiction. 2012;107 Suppl 1:45-52.

19. Winklbaur B, Baewert A, Jagsch R, et al. Association between prenatal exposure and outcome of neonates born to opioid-maintained mothers. Eur Addict Res. 2009;15:150-156.

20. Wachman EM, Newby PK, Vreeland J, et al. The relationship between maternal opioid agonists and psychiatric medications on length of hospitalization for neonatal abstinence syndrome. J Addict Med. 2011;5: 293-299.

21. Cleary BJ, Donnell J, Strawbridge J, et al. Methadone dose and neonatal abstinence syndrome - systematic review and meta-analysis. Addiction. 2010;105:2071-2084.

22. Jones HE, Heil SH, Baewert A, et al. Buprenorphine treatment of opioiddependent pregnant women: a comprehensive review. Addiction. 2012; 107 Suppl 1:S5-S27.

23. Jones HE, Dengler E, Garrison A, et al. Neonatal outcomes and their relationship to maternal buprenorphine dose during pregnancy. Drug Alcohol Depend. 2014;134:414-417.

24. Dysart K, Hsieh H-C, Kaltenbach K, Greenspan JS. Sequela of preterm versus term infants born to mothers on a methadone maintenance program: differential course of neonatal abstinence syndrome. J Perinat Med. 2007;35:344-346.

25. Doberczak TM, Kandall SR, Wilets I. Neonatal opiate abstinence syndrome in term and preterm infants. J Pediatr. 1991;118:933-937.

26. Jansson LM, Velez M. Neonatal abstinence syndrome. Curr Opin Pediatr. 2012;24:252-258.

27. Finnegan LP, Kron RE, Connaughton JF, Emich JP. Assessment and treatment of abstinence in the infant of the drug-dependent mother. Int J Clin Pharmacol. 1975;12:19-32.

28. Finnegan LP, Kaltenbach K. Neonatal abstinence syndrome. In: Hoekelman RA, Friedman SB, Nelson N, Seidel HM, editors. Primary Pediatric Care. 2nd ed. St Louis MO, USA: Mosby-Yearbook Inc.; 1992.

29. Sarkar S, Donn SM. Management of neonatal abstinence syndrome in neonatal intensive care units: a national survey. J Perinatol. 2006;26:15-17.
30. O'Grady MJ, Hopewell J, White MJ. Management of neonatal abstinence syndrome: a national survey and review of practice. Arch Dis Child Fetal Neonatal Ed. 2009;94:F249-F252.

31. Zimmerman-Baer U, Notzli U, Rentsch K, Bucher HU. Finnegan neonatal abstinence scoring system: normal values for first 3 days and weeks 5-6 in non-addicted infants. Addiction. 2010;105:524-528.

32. Lipsitz PJ. Proposed narcotic withdrawal score for use with newborn infants. A pragmatic evaluation of its efficacy. Clin Pediatr (Phila). 1975;14:592-594.

33. Jansson LM, Velez M, Harrow C. The opioid-exposed newborn. J Opioid Manag. 2009;5:47-55.

34. Johnson K, Gerada C, Greenough A. Treatment of neonatal abstinence syndrome. Arch Dis Child Fetal Neonatal Ed. 2003;88:F2-F5.

35. Kraft WK, van den Anker JN. Pharmacologic management of the opioid neonatal abstinence syndrome. Paper 39. Department of Pharmacology and Experimental Therapeutics Faculty Papers; 2012. Available from: http://jdc.jefferson.edu/cgi/viewcontent. cgi? article=1038\&context=petfp. Accessed February 14, 2014.

36. Osborn DA, Jefffery HE, Cole M. Opiate treatment for opiate withdrawal in newborn infants. Cochrane Database Syst Rev. 2010;10:CD002059.

37. Lainwala S, Brown ER, Weinschenk NP, Blackwell MT, Hagadorn JI. A retrospective study of length of hospital stay in infants treated for neonatal abstinence syndrome with methadone versus oral morphine preparations. Adv Neonatal Care. 2005;5:265-272.

38. Kraft WK, Gibson E, Dysart K, et al. Sublingual buprenorphine for treatment of neonatal abstinence syndrome: a randomized trial. Pediatrics. 2008;122:e601-e607.

39. Kraft WK, Dysart K, Greenspan JS, Gibson E, Kaltenbach K, Ehrlich ME. Revised dose schema of sublingual buprenorphine in the treatment of the neonatal opioid abstinence syndrome. Addiction. 2010;106: 574-580.

40. Agthe AG, Kim GR, Mathias KB, et al. Clonidine as an adjunct therapy to opioids for neonatal abstinence syndrome: a randomized, controlled trial. Pediatrics. 2009;123:e849-e856.

41. Osborn DA, Jeffery HE, Cole MJ. Sedatives for opiate withdrawal in newborn infants. Cochrane Database Syst Rev. 2005;3:CD002053.

42. Surran B, Visintainer P, Chamberlain S, Kopcza K, Shah B, Singh R. Efficacy of clonidine versus phenobarbital in reducing neonatal morphine sulfate therapy days for neonatal abstinence syndrome. A prospective randomized clinical trial. J Perinatol. 2013;33:954-959.

43. Oei J, Feller JM, Lui K. Coordinated outpatient care of the narcoticdependent infant. J Paediatr Child Health. 2001;37:266-270.

44. Backes CH, Backes CR, Gardner D, Nankervis CA, Giannone PJ, Cordero L. Neonatal abstinence syndrome: transitioning methadonetreated infants from an inpatient to an outpatient setting. J Perinatol. 2012;32:425-430

45. McLemore GL, Lewis T, Jones CH, Gauda EB. Novel pharmacotherapeutic strategies for treatment of opioid-induced neonatal abstinence syndrome. Semin Fetal Neonatal Med. 2013;18:35-41.

46. D'Apolito K. Comparison of a rocking bed and standard bed for decreasing withdrawal symptoms in drug-exposed infants. Am J Matern Child Nurs. 1999;24:138-144.

47. Beauman SS. Identification and management of neonatal abstinence syndrome. J Infus Nurs. 2005;28:159-167.

48. Abdel-Latif ME, Pinner J, Clews S, Cooke F, Lui K, Oei J. Effects of breast milk on the severity and outcome of neonatal abstinence syndrome among infants of drug-dependent mothers. Pediatrics. 2006;117:e1163-e1169.

49. McQueen KA, Murphy-Oikonen J, Gerlach K, Montelpare W. The impact of infant feeding method on neonatal abstinence scores of methadone-exposed infants. Adv Neonatal Care. 2011;11:282-290.

50. Jansson LM, Choo R, Velez ML, et al. Methadone maintenance and breastfeeding in the neonatal period. Pediatrics. 2008;121:106-114.

51. Pritham UA, Paul JA, Hayes MJ. Opioid dependency in pregnancy and length of stay for neonatal abstinence syndrome. J Obstet Gynecol Neonatal Nurs. 2012;41:180-190. 
52. Marquet P, Chevrel J, Lavignasse P, Merle L, Lachatre G. Buprenorphine withdrawal syndrome in a newborn. Clin Pharmacol Ther. 1997;62:569-571.

53. Grimm D, Pauly E, Poschl J, Linderkamp O, Skopp G. Buprenorphine and norbuprenorphine concentrations in human breast milk samples determined by liquid chromatography - tandem mass spectrometry. Ther Drug Monit. 2005;27:526-530.

54. Lindemalm S, Nydert P, Svensson JO, Stahle L, Sarman I. Transfer of buprenorphine into breast milk and calculation of infant drug use. J Hum Lact. 2009;25:199-205.

55. Ilett KF, Hackett P, Gower S, Doherty DA, Hamilton D, Bartu AE. Estimated dose exposure of the neonate to buprenorphine and its metabolite norbuprenorphine via breastmilk during maternal buprenorphine substitution treatment. Breastfeed Med. 2012;7:269-274.

56. O'Connor A, Alto W, Musgrave K, et al. Observational study of buprenorphine treatment of opioid-dependent pregnant women in a family medicine residency: reports on maternal and infant outcomes. J Am Board Fam Med. 2011;24:194-201.

57. Madden JD, Payne TF, Miller S. Maternal cocaine abuse and effect on the newborn. Pediatrics. 1986;77:209-211.

58. Oro AS, Dixon SD. Perinatal cocaine and methamphetamine exposure: maternal and neonatal outcomes. J Pediatr. 1987;111:571-578.

59. Doberczak TM, Shanzer S, Senie RT, Kandall SR. Neonatal neurologic and electroencephalographic effects of intrauterine cocaine exposure. J Pediatr. 1988;113:354-358.

60. Richardson GA, Day NL. Maternal and neonatal effects of moderate cocaine use during pregnancy. Neurotoxicol Teratol. 1991;13:455-460.

61. Richardson GA, Hamel SC, Goldschmidt L, Day NL. The effects of prenatal cocaine use on neonatal neurobehavioral status. Neurotoxicol Teratol. 1996;18:519-528.

62. Bandstra ES, Morrow CE, Mansoor E, Accornero VH. Prenatal drug exposure: infant and toddler outcomes. J Addict Dis. 2010;29: 245-258.

63. Behnke M, Smith VC. Prenatal substance abuse: short- and long-term effects on the exposed fetus. Pediatrics. 2013;131:e1009-e1024.

64. Eyler FD, Behnke M, Wilson Garvan C, Stewart Woods N, Wobie K, Conlon M. Newborn evaluations of toxicity and withdrawal related to prenatal cocaine exposure. Neurotoxicol Teratol. 2001;23:399-411.

65. Bauer CR, Langer JC, Shankaran S, et al. Acute neonatal effects of cocaine exposure during pregnancy. Arch Pediatr Adolesc Med. 2005; 159:824-834.

66. Fried PA, Makin JE. Neonatal behavioural correlates of prenatal exposure to marihuana, cigarettes and alcohol in a low risk population. Neurotoxicol Teratol. 1987;9:1-7.

67. Fried PA, Watkinson B, Dilllon RF. Neonatal neurological status in a low-risk population after prenatal exposure to cigarettes, marijuana, and alcohol. J Dev Behav Pediatr. 1987;8:318-326.

68. Lester BM, Dreher M. Effects of marijuana use during pregnancy on newborn cry. Child Dev. 1989;60:765-771.

69. Scher MS, Richardson GA, Coble PA, Day NL, Stoffer DS. The effects of prenatal alcohol and marihuana exposure: disturbances in neonatal sleep cycling and arousal. Pediatr Res. 1988;24:101-105.

70. Richardson GA, Day NL, Taylor PM. The effect of prenatal alcohol, marijuana, and tobacco exposure on neonatal behavior. Infant Behav Dev. 1989;12:199-209.
71. Ross LE, McLean LM. Anxiety disorders during pregnancy and the postpartum period: a systematic review. J Clin Psychiatry. 2006;67: 1285-1298.

72. Iqbal MM, Sobhan T, Ryals T. Effects of commonly used benzodiazepines on the fetus, the neonate, and the nursing infant. Psychiatr Serv. 2002;53:39-49.

73. Laegreid L. Potential hazards of benzodiazepines for the fetus. Acta Obstet Gynecol Scand. 1992;71:655-656.

74. Laegreid L, Hagberg G, Lundberg A. The effect of benzodiazepines on the fetus and the newborn. Neuropediatrics. 1992;23:18-23.

75. Rementeria JL, Bhatt K. Withdrawal symptoms in neonates from intrauterine exposure to diazepam. J Pediatr. 1977;90:123-126.

76. McAuley DM, O’Neill MP, Moore J, Dundee JW. Lorazepam premedication for labour. BJOG. 1982;89:149-154.

77. Gillberg C. "Floppy infant syndrome" and maternal diazepam. Lancet. 1977;2:244.

78. Calderon-Margalit R, Qiu C, Ornoy A, Siscovick DS, Williams MA. Risk of preterm delivery and other adverse perinatal outcomes in relation to maternal use of psychotropic medications during pregnancy. Am J Obstet Gynecol. 2009;201:579:e1-e8.

79. Gavin NI, Gaynes BN, Lohr KN, Meltzer-Brody S, Gartlehner G, Swinson T. Perinatal depression: a systematic review of prevalence and incidence. Obstet Gynecol. 2005;106(5 Part 1):1071-1083.

80. Huybrechts KF, Palmsten K, Mogun H, et al. National trends in antidepressant medication treatment among publicly insured pregnant women. Gen Hosp Psychiatry. 2013;35:265-271.

81. Meltzer-Brody S, Boschloo L, Jones I, Sullivan PF, Penninx BW. The EPDS-Lifetime: assessment of lifetime prevalence and risk factors for perinatal depression in a large cohort of depressed women. Arch Womens Ment Health. 2013;16:465-473.

82. Andrade SE, Raebel MA, Brown J, et al. Use of antidepressant medications during pregnancy: a multisite study. Am J Obstet Gynecol. 2008;198:194.e1-194.e5.

83. Alwan S, Reefhuis J, Rasmussen SA, Friedman JM; National Birth Defects Prevention Study. Patterns of antidepressant medication use among pregnant women in a United States population. J Clin Pharmacol. 2011;51:264-270.

84. Udechuku A, Nguyen R, Hil R, Szego K. Antidepressants in pregnancy: a systematic review. Aust N Z J Psychiatry. 2010;44:978-996.

85. Koren G, Nordeng H. Antidepressant use during pregnancy: the benefitrisk ratio. Am J Obstet Gynecol. 2012;44:157-163.

86. Hayes RM, Wu P, Shelton RC, et al. Maternal antidepressant use and adverse outcomes: a cohort study of 228,876 pregnancies. Am J Obstet Gynecol. 2012;207:49.e1-49.e9.

87. Jong GW, Einarson T, Koren G, Einarson A. Antidepressant use in pregnancy and persistent pulmonary hypertension of the newborn (PPHN): a systematic review. Reprod Toxicol. 2012;34:293-297.

88. Chambers CD, Hernandez-Diaz S, Van Marter LJ, et al. Selective serotonin-reuptake inhibitors and risk of persistent pulmonary hypertension of the newborn. $N$ Engl J Med. 2006;354:579-587.

89. Andrade SE, McPhillips H, Loren D, et al. Antidepressant medication use and risk of persistent pulmonary hypertension of the newborn. Pharmacoepidemiol Drug Saf. 2009;18:246-252.

90. Jefferies AL. Selective serotonin reuptake inhibitors in pregnancy and infant outcomes. Paediatr Child Health. 2011;16:562-563.
Research and Reports in Neonatology

\section{Publish your work in this journal}

Research and Reports in Neonatology is an international, peer-reviewed, open access journal publishing original research, reports, editorials, reviews and commentaries on neonatal health. The manuscript management system is completely online and includes a very quick and fair peer-review system. Visit http://www.dovepress.com/testimonials.php to read real quotes from published authors. 